\title{
DINÂMICA POPULACIONAL DO PARASITOIDE DE OVOS Erythmelus tingitiphagus (HYMENOPTERA: MYMARIDAE) EM CLONE DE SERINGUEIRA, EM ITIQUIRA, MT ${ }^{1}$
}

\author{
Rodrigo Souza Santos² e Joaquim Manoel da Silva ${ }^{3}$
}

\begin{abstract}
RESUMO - O percevejo-de-renda Leptopharsa heveae Drake \& Poor (Hemiptera: Tingidae) é uma das mais importantes pragas da heveicultura no Brasil, principalmente nas regiões Sudeste e Centro-Oeste. Devido ao seu hábito sugador, na face abaxial das folhas, esta praga leva à senescência precoce das mesmas e a reduções na produção de látex em até 30\%. Dentre os inimigos naturais de L. heveae está o parasitoide de ovos Erythmelus tingitiphagus (Soares) (Hymenoptera: Mymaridae), regulando suas populações em condições naturais. O objetivo deste estudo foi verificar a dinâmica populacional deste parasitoide, bem como correlacioná-la com os fatores meteorológicos temperatura e pluviosidade, em plantio comercial de seringueira do clone PB 217, em Itiquira, MT. Semanalmente foram coletadas quatro folhas maduras por árvore, no terço inferior da copa de 40 árvores, totalizando 160 folhas por amostragem, no período de agosto de 2006 a janeiro de 2007. Houve correlação positiva entre a dinâmica populacional e os fatores meteorológicos, sendo o pico populacional do parasitoide observado no mês de novembro e declinando até janeiro na área estudada.
\end{abstract}

Palavras-chave: Controle biológico, Hevea brasiliensis, Flutuação populacional.

\section{POPULATION DYNAMIC OF EGG PARASITOID Erythmelus tingitiphagus (HYMENOPTERA: MYMARIDAE) IN RUBBER TREE CLONE IN ITIQUIRA, MATO GROSSO STATE, BRAZIL}

\begin{abstract}
The lace bug Leptopharsa heveae Drake \& Poor (Hemiptera: Tingidae) is one of the most import heveiculture pests in Brazil, mainly in the southeast and central-west regions. Due to its sucking habit on the abaxial surface, this pest causes precocious senescence in leaves, and leads to the reduction in latex production in up to $30 \%$ of them. Among the natural enemies of $\boldsymbol{L}$. heveae is the egg parasitoid Erythmelus tingitiphagus (Soares) (Hymenoptera: Mymaridae), regulating populations of L. heveae in natural conditions. The objective of this work was to verify the population dynamics of this parasitoid, as well as to correlate it with meteorological factors such as temperature and rainfall, in a commercial plantation of rubber trees of the PB 217 clone, in Itiquira, in the Mato Grosso state, Brazil. Four mature leaves were collected weekly, on the lower stratum of canopy of PB 217 clone in 40 trees, totaling 160 leaves in each sampling, from August, 2006 to January, 2007. There was a positive correlation between population dynamics and meteorological factors, the population peak of the parasitoid being observed in November and declining until January in the study area.
\end{abstract}

Keywords: Biological control, Hevea brasiliensis, Population flotation.

\footnotetext{
${ }^{1}$ Recebido em 10.10.2010 aceito para publicação em 05.04.2013.

${ }^{2}$ Laboratório de Entomologia, Embrapa Acre. E-mail:<rodrigo.s.santos@embrapa.br>.

${ }^{3}$ Universidade do Estado do Mato Grosso (UNEMAT) - Campus de Nova Xavantina. E-mail:<joaquim.manoel@ gmail.com>.
} 


\section{INTRODUÇÃO}

A seringueira pertence ao gênero Hevea Aubl. (Euphorbiaceae), originário da região Amazônica, que inclui outros gêneros importantes de culturas tropicais, como Ricinus L. (mamona) e Manihot Mill. (mandioca). O gênero Hevea engloba 39 espécies e apresenta grande variabilidade morfológica, variando de florestas altas até arbustos, com 11 espécies encontradas no Brasil (GONÇALVES et al., 1997; TROPICOS, 2013). Hevea brasiliensis (Willd. ex Adr. de Juss.) Müell. Arg. é considerada a mais importante, com superior qualidade de látex e alta produtividade (GONÇALVES et al., 2002).

A partir do seu hábitat, a seringueira passou a ser cultivada em sistema de monocultura em extensas áreas e favorece a adaptação de insetos que se tornam problema (ALTIERI, 1994), interferindo na fisiologia da planta e na produção de látex. Uma alternativa para a monocultura seria o plantio de seringais em sistema de consórcio com outras espécies vegetais nas entrelinhas, gerando renda ao produtor antes do início do processo de sangria (MAY et al., 1999), além da diversificação do agroecossistema, bem como pela possibilidade de comercialização de créditos de carbono (COTTA et al., 2006).

Um complexo de pragas está associado à cultura da seringueira no Brasil, destacando-se os ácaros Calacarus heveae Feres, Phyllocoptruta seringueirae Feres (Acari: Eriophyidae) e Tenuipalpus heveae Baker (Acari: Tenuipalpidae) (FERES et al., 2002; BELLINI et al., 2005; FERLA; MORAES, 2008), as formigascortadeiras dos gêneros Atta Fabricius e Acromyrmex Mayr (ANJOS et al., 2008) e o percevejo-de-renda Leptopharsa heveae Drake \& Poor (Hemiptera: Tingidae) (MOREIRA, 1986; SANTOS; FREITAS, 2008a). Este último forma grandes colônias na face abaxial das folhas, de onde suga a seiva elaborada, destruindo o parênquima clorofiliano e, consequentemente, promovendo perda de área fotossintetizante e favorecendo a entrada de microrganismos secundários (MOREIRA, 1986; VENDRAMIM, 1992; SANTOS; FREITAS, 2008a), sendo considerada praga-chave em seringais de cultivo no Centro-Oeste do Brasil. O ataque em altas infestações pode provocar redução de até $28 \%$ no crescimento em altura e de até $44,5 \%$ no diâmetro do colo das plantas, em mudas de seringueira em viveiros (MOREIRA, 1986) ou, ainda, queda na produção de látex em até $30 \%$ (TANZINI; LARA, 1998).

Revista Árvore, Viçosa-MG, v.37, n.2, p.237-244, 2013
Entre os principais inimigos naturais de L. heveae, encontram-se os crisopídeos (Neuroptera: Chrysopidae) (SCOMPARIN, 1997), o fungo entomopatogênico Sporothrix insectorum (Hoog \& Evans) (TANZINI, 2002) e o parasitoide de ovos Erythmelus tingitiphagus (Soares) (Hymenoptera: Mymaridae) (Figura 1) (COSTA et al., 2003; SANTOS; FREITAS, 2008ab).

Estudos de dinâmica populacional de insetos-praga e de seus inimigos naturais são fundamentais para o conhecimento da época de maior ocorrência de ambos, proporcionando, assim, o estabelecimento de programas de controle eficientes e racionais e viabilizando o planejamento das estratégias de manejo mais eficazes (GINGRICH, 1993; RONCHI-TELES; SILVA, 2005). Estudos dessa natureza ainda não foram realizados para E. tingitiphagus em talhões de seringueira no Brasil. Nesse sentido, este estudo objetivou conhecer a dinâmica populacional e a influência da temperatura e pluviosidade na população de E. tingitiphagus, em plantio comercial de seringueira do clone PB 217, na fazenda da empresa "Plantações E. Michelin Ltda.", no Município de Itiquira, MT.

Fonte: Rodrigo Souza Santos. Source: Rodrigo Souza Santos.

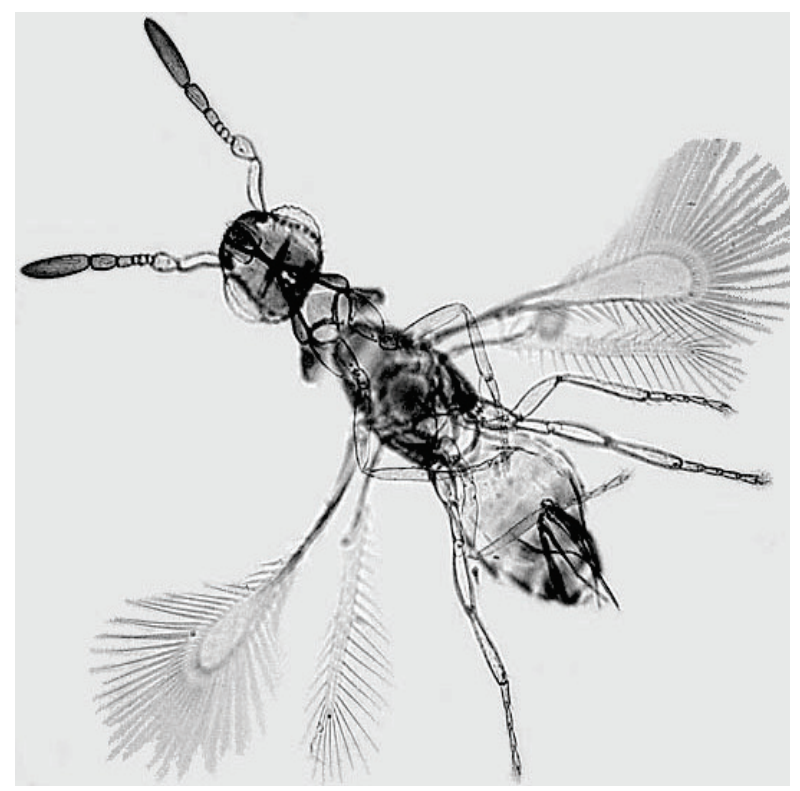

Figura 1 - Fêmea adulta de Erythmelus tingitiphagus (Hymenoptera: Mymaridae).

Figure 1 -Erythmelus tingitiphagus adult female (Hymenoptera: Mymaridae). 


\section{MATERIAL E MÉTODOS}

O estudo foi conduzido de agosto de 2006 a janeiro de 2007 (período de ocorrência de L. heveae em campo) na fazenda da empresa "Plantações E. Michelin Ltda.", Município de Itiquira, MT (17²2’23”S, 5444'23”W), em um talhão de seringueira do clone PB 217 (talhão 310B), com aproximadamente 6,32 ha, árvores com 21 anos de idade, altura média de 11 a $13 \mathrm{~m}$ e cultivadas no espaçamento de $2,5 \times 8 \mathrm{~m}$. As plantas receberam tratamento convencional com produtos fitossanitários com os fungos Paecilomyces sp. e, ou, S. insectorum adicionados na calda durante o período de realização desta pesquisa. O clone PB 217 foi escolhido pela facilidade de coleta das folhas, pela uniformidade das árvores no talhão, por sua boa produtividade de látex e pelo nível de ataque de L. heveae neste clone.

Foram realizadas 23 coletas aleatórias e semanais, de folhas maduras (completamente expandidas) com sintomas de ataque de $L$. heveae, no terço inferior da copa de 40 árvores (10 árvores em cada lado do talhão, para uma amostragem mais abrangente), com o auxílio de podão com cabo telescópico, totalizando 160 folhas por amostragem. As folhas foram acondicionadas em sacos de papel identificados e levadas ao laboratório, localizado na usina de beneficiamento de látex da empresa. Segundo Cividanes et al. (2004), ninfas e adultos de L. heveae se distribuem uniformemente nos diferentes estratos da planta, não comprometendo a amostragem vertical.

Em laboratório foi selecionado, aleatoriamente, um folíolo por folha, totalizando 160 folíolos por ensaio. Estes foram submetidos à lavagem, com suaves pinceladas, em solução de hipoclorito de sódio (1,5\%) durante 2 min, para remoção de impurezas e ovos de outros insetos que pudessem se encontrar na superfície dos folíolos. Estes foram enxaguados em água destilada por 2 min e mantidos em papel absorvente até a secagem, seguindo-se a metodologia adaptada de Costa et al. (2003). O quantitativo de ovos endofíticos de L. heveae não foi contabilizado nos ensaios.

Os pecíolos de cada folíolo foram inseridos em tubos plásticos (ponteira de micropipeta com a extremidade afilada lacrada) contendo água destilada e com sua abertura vedada com Parafilm $\mathbf{M}^{\circledR}$ para evitar o seu escoamento (Figura 2). Esse material (tubos + folíolos) foi acondicionado em sacos plásticos $(12 \times 30 \mathrm{~cm})$ codificados, inflados com um compressor de ar, selados com o auxílio de uma seladora elétrica e pendurados em varais, com o auxílio de prendedores, no interior de uma sala climatizada a $25 \pm 1{ }^{\circ} \mathrm{C}$ e fotofase de $12 \mathrm{~h}$.

As avaliações foram realizadas sete dias após a coleta, observando-se os sacos plásticos e folíolos sob microscópio estereoscópico e contabilizando o número de parasitoides emergidos. Com o auxílio de um instrumento pontiagudo, os ovos foram dissecados para selecionar os que não tiveram emergência. Os dados meteorológicos foram fornecidos pela estação meteorológica da empresa "Plantações E. Michelin Ltda." (17 $\left.37^{\circ} 24^{\prime \prime S}, 54^{\circ} 73^{\prime} 87^{\prime \prime} \mathrm{W}\right)$, localizada cerca de 1.000 $\mathrm{m}$ do talhão de seringueira estudado.

Os dados foram submetidos ao teste de correlação linear de Pearson (r) para analisar separadamente a influência dos fatores meteorológicos (temperatura e pluviosidade) na dinâmica populacional de E. tingitiphagus, e a significância foi testada com a utilização do teste “t”, de Student, a 10\% de probabilidade. Foi realizada uma análise de variância de regressão múltipla, com o objetivo de analisar as variáveis meteorológicas em conjunto, utilizando o logaritmo do número de parasitoides, segundo o modelo ajustado: $\log _{\left(\mathrm{n}^{\circ} \text { de parasitoides }\right.}=\mathrm{b} 0+\mathrm{b} 1 *$ temperatura $+\mathrm{b} 2 *$ pluviosidade + b3*temperatura* pluviosidade+erro.

Nas correlações, os dados referentes ao parasitoide foram correlacionados com o programa $\mathrm{R}$, versão 2.11.0 (2010).

Os parasitoides obtidos foram preservados em frascos de vidro identificados contendo etanol (80\%) e enviados ao taxonomista Dr. Valmir Antonio Costa (Instituto Biológico/APTA, Campinas, SP), para identificação.

Fonte: Rodrigo Souza Santos.

Source: Rodrigo Souza Santos.

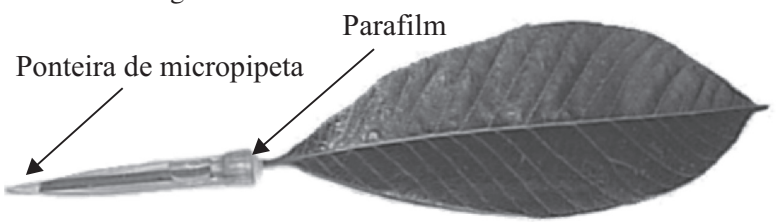

Figura 2 - Folíolo de seringueira (Hevea brasiliensis) com o pecíolo no interior de tubo plástico contendo água destilada e vedado com Parafilm $\mathbf{M}^{\circledR}$.

Figure 2-Rubber tree (Hevea brasiliensis) foliole with petiole inserted into plastic tube containing distilled water and sealed with Parafilm $M^{\circledR}$.

Revista Árvore, Viçosa-MG, v.37, n.2, p.237-244, 2013 


\section{RESULTADOS}

Dos 3.680 folíolos analisados foram obtidos 2.946 parasitoides, sendo 2.921 espécimes de E. tingitiphagus numa razão de 0,79 parasitoide por folíolo e com média de 127 parasitoides por amostragem. As maiores relações parasitoide/folíolo foram observadas no mês de novembro de 2006, atingindo 2,72 parasitoides por folíolo, sendo a menor observada no mês de agosto de 2006, quando não se obteve nenhum exemplar do parasitoide (Figura 3). No mês de novembro de 2006, houve uma concentração de $74,4 \%$ do total de parasitoides obtidos durante todo o período do estudo. Além de E. tingitiphagus, foram obtidos 25 espécimes de parasitoides de outras duas espécies: Schizophragma bicolor (Dozier) (Hymenoptera: Mymaridae) e Epoligosita mexicana Viggiani (Hymenoptera: Trichogrammatidae). Não há relatos na literatura sobre a associação dessas espécies com L. heveae e, dessa forma, não foram consideradas nesta pesquisa.

A população de E. tingitiphagus aumentou gradualmente a partir de outubro de 2006, atingindo o pico populacional em meados de novembro de 2006 e declinando até janeiro de 2007 (Figura 2). Foi observado aumento expressivo da população do parasitoide a partir do início do período chuvoso na região, que ocorreu na segunda semana de outubro de 2006.

A média geral das temperaturas médias semanais foi de $25,1^{\circ} \mathrm{C}$, variando de 22,7 a $27,2{ }^{\circ} \mathrm{C}$ na terceira semana de agosto de 2006 e nas duas primeiras semanas de novembro/2006, respectivamente. As chuvas iniciaram-se em setembro de 2006, apresentando os maiores índices pluviométricos em novembro e dezembro de 2006 (Figura 3).

Houve correlação positiva entre o número de parasitoides e a temperatura média semanal, apresentando coeficiente de correlação estatisticamente positivo. No entanto, as correlações entre o número de parasitoides e a pluviosidade e a temperatura e pluviosidade não foram estatisticamente significativas (Tabela 1). Pela análise de regressão múltipla, os fatores meteorológicos temperatura, pluviosidade e temperatura versus pluviosidade apresentaram significância estatística com $\mathrm{F}_{1,19}=6,57 ; \mathrm{p}=0,02, \mathrm{~F}_{1,19}=5,88 ; \mathrm{p}=0,03 \mathrm{eF}_{1,19}=3,64 ; \mathrm{e} \mathrm{p}=0,07$, respectivamente (Tabela 2 ). $\mathrm{O}$ valor do coeficiente de determinação $\left(\mathrm{r}^{2}-\right)$ foi de $0,46, \operatorname{com~}_{3,19}=5,36$ e $\mathrm{p}=0,007$, ou seja, $46 \%$ da dinâmica populacional do parasitoide pode ser explicada pela variação da temperatura, pluviosidade e interação desses fatores meteorológicos.

\section{DISCUSSÃO}

A obtenção de espécimes não associados a L. heveae deveu-se à metodologia utilizada, na qual se eliminavam as posturas superficiais, nas faces abaxiais e adaxiais dos folíolos, mas não as posturas endofíticas, como as de L. heveae e de outros insetos com essa estratégia de oviposição. Costa et al. (2003) observaram E. tingitiphagus parasitando ovos de L. heveae em

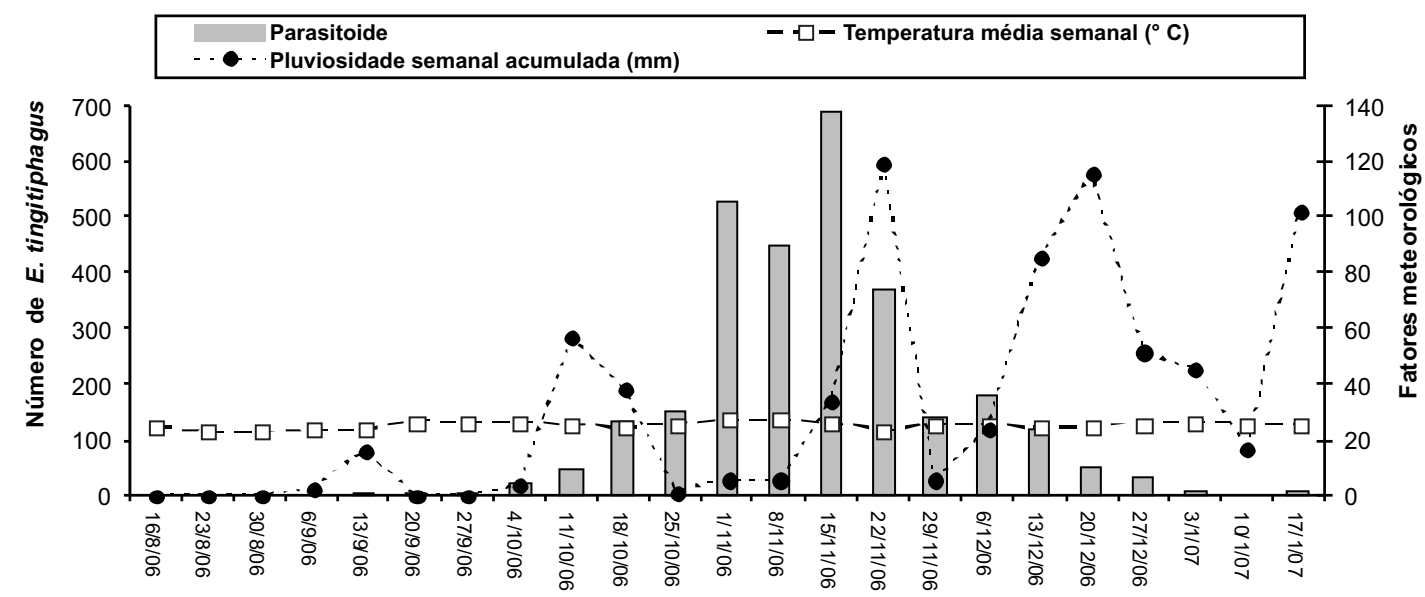

Figura 3 - Dinâmica populacional de Erythmelus tingitiphagus, temperatura média semanal $\left({ }^{\circ} \mathrm{C}\right)$ e pluviosidade semanal acumulada (mm) em área cultivada com o clone de seringueira PB 217, de agosto de 2006 a janeiro de 2007, em Itiquira, MT.

Figure 3 - Population dynamics of Erythmelus tingitiphagus, weekly average temperature $\left({ }^{\circ} \mathrm{C}\right)$ and accumulated weekly rainfall ( $\mathrm{mm}$ ) in cultivated area with the PB 217 rubber tree clone, from August 2006 to January 2007 in Itiquira, MT.

Revista Árvore, Viçosa-MG, v.37, n.2, p.237-244, 2013 
Tabela 1 - Coeficiente de correlação de Pearson (r) e significância (t) entre o número de Erythmelus tingitiphagus e os fatores meteorológicos temperatura $\left({ }^{\circ} \mathrm{C}\right)$ e pluviosidade $(\mathrm{mm})$, no período de agosto de 2006 a janeiro de 2007, em Itiquira, MT.

Table 1 -Pearson's correlation coefficient $(r)$ and significance $(t)$ between the number of Erythmelus tingitiphagus and meteorological factors temperature $\left({ }^{\circ} \mathrm{C}\right)$ and rainfall $(\mathrm{mm})$, from August 2006 to January 2007 in Itiquira, MT.

\begin{tabular}{lccc}
\hline Correlações & $\mathrm{r}$ & g. 1. & $\mathrm{t}$ \\
\hline Parasitoide X Temperatura $\left({ }^{\circ} \mathrm{C}\right)$ & 0,379 & 21 & $0,075^{* *}$ \\
Parasitoide X Pluviosidade $(\mathrm{mm})$ & 0,072 & 21 & $0,743^{\mathrm{ns}}$ \\
Temperatura $\left({ }^{\circ} \mathrm{C}\right)$ X Pluviosidade $(\mathrm{mm})$ & $-0,171$ & 21 & $0,435^{\mathrm{ns}}$ \\
\hline
\end{tabular}

g. 1. - graus de liberdade; ns não significativo e ${ }^{* *}$ significativo $(P \geq 0,10)$.

folhas do clone PB 235 em Pindorama, SP. A espécie Epoligosita duliniae Livingstone e Yacoob (Hymenoptera: Trichogrammatidae), que ocorre na Índia, está associada ao tingídeo Dulinius conchatus Distant (Hemiptera: Tingidae) (LIVINGSTONE; YACOOB, 1983). Dessa forma, há dentro do gênero Epoligosita Girault, pelo menos, uma espécie que parasita Tingidae, necessitando da confirmação da associação entre E. mexicana e L. heveae. Mesmo que essa associação tivesse sido constatada, é importante salientar que, devido à grande quantidade de E. tingitiphagus obtidos em relação a $E$. mexicana, evidenciou-se que a competição entre essas espécies por hospedeiros (ovos) seja irrelevante para E. tingitiphagus.

A quantidade insignificante de folhas maduras de seringueiras no mês de agosto de 2006 pode ter sido o principal fator responsável pela ausência de parasitoides nesse período, pois a ocorrência de L. heveae infestando o talhão era insignificante. De acordo com Santos (2007), a fenologia do clone PB 217 na região, seu reenfolhamento ocorre no início de agosto, apresentando folhas maduras em meados de setembro e tendo sido observado maior infestação de L. heveae em folhas maduras, em detrimento das novas e de idade intermediária, o que sugere uma preferência de alimentação e oviposição de L. heveae em folhas maduras. Dessa forma, com maior quantidade de folhas maduras e de hospedeiros disponíveis (ovos), houve aumento progressivo da população do parasitoide, numa clara relação de dependência do parasitoide em relação à densidade populacional do seu hospedeiro.

Segundo Thaler (1999), substâncias produzidas pela planta devido às injúrias causadas pelas pragas podem atuar como sinalizadores químicos para os parasitoides. Dessa forma, o aumento na população do inimigo natural não depende somente do nível populacional da praga, mas, também, das injúrias causadas por ela e dos sinais químicos voláteis (semioquímicos) liberados pela planta, todos esses fatores atuando sinergicamente nas populações da praga e do inimigo natural.

O pico populacional de E. tingitiphagus, na área do estudo, ocorreu no mês de novembro de 2006 e declinou a partir de dezembro até janeiro de 2007 (Figura 3). Esse resultado corrobora os obtidos por Santos e Freitas (2008a), em que o número médio de ovos de L. heveae por folíolo e de E. tingitiphagus aumentaram no mês de novembro para os clones RRIM 600, PR 255, PB 235 e PB 217, declinando sensivelmente a partir de dezembro até janeiro, em Itiquira, MT. O declínio populacional do parasitoide deve-se, principalmente, à diminuição da população de L. heveae na área de realização desta pesquisa nos meses de dezembro e janeiro.

Conforme a Tabela 1, a temperatura tendeu a ser o principal fator meteorológico influenciando a população do parasitoide na área. A temperatura é um dos fatores ambientais que interferem diretamente no desenvolvimento da população de insetos, sendo a temperatura ótima para esse evento em torno de $25^{\circ} \mathrm{C}$, correspondendo ao desenvolvimento mais acelerado e com maior quantidade de descendentes (RODRIGUES, 2004). A média da temperatura na região foi registrada em torno de $25,1^{\circ} \mathrm{C}$, estando dentro do limite ótimo de desenvolvimento, tanto para as populações de $L$. heveae quanto para as do parasitoide. O pico populacional de $L$. heveae ocorreu nas duas primeiras semanas do mês de novembro de 2006, período em que foram observadas as maiores médias de temperatura $\left(27,2^{\circ} \mathrm{C}\right)$ (Figura 2$)$, além do aumento populacional de L. heveae no talhão e, consequentemente, aumentando a oferta de hospedeiros disponíveis, conforme observado no decorrer da pesquisa.

Revista Árvore, Viçosa-MG, v.37, n.2, p.237-244, 2013 
O padrão de sazonalidade de insetos tropicais tem sido, muitas vezes, correlacionado com a precipitação, com os picos de abundância ocorrendo na estação chuvosa(WOLDA, 1980, 1988). Foi verificado que grande aumento populacional do parasitoide ocorreu logo após o início da estação chuvosa na região (outubro de 2006), coincidindo com a elevação do índice pluviométrico (Figura 3).

A interação dos fatores temperatura e pluviosidade influenciou em aproximadamente $46 \%$ na dinâmica populacional (Tabela 2), com a ressalva de que outros fatores, como o potencial biótico do inimigo natural, a disponibilidade de hospedeiros, a resistência ambiental e o uso de controle fitossanitário, também podem ter influenciado.

A aplicação de produtos fitossanitários no talhão estudado pareceu não ter proporcionado forte impacto negativo na população de E. tingitiphagus. Provavelmente, por ser um parasitoide idiobionte de ovos endofíticos, os ovos parasitados encontravam-se protegidos contra a ação da nebulização do produto fitossanitário, condicionando a emergência dos parasitoides adultos e uma rápida recolonização da área, após as aplicações. Outro fator relevante seria o modo da aplicação dos produtos fitossanitários no talhão (apenas no foco da infestação de $L$. heveae e não em todo o talhão), diminuindo o impacto negativo das pulverizações sobre os parasitoides adultos presentes na área e no momento das aplicações, além de diminuir os gastos com tal procedimento.

Estudos a respeito da interação entre plantaparasitoide sobre a criação massal em laboratório e

Tabela 2 - Análise dos coeficientes da regressão múltipla entre o número de Erythmelus tingitiphagus e os fatores meteorológicos temperatura $\left({ }^{\circ} \mathrm{C}\right) \mathrm{e}$ pluviosidade $(\mathrm{mm})$, no período de agosto de 2006 a janeiro de 2007, em Itiquira, MT.

Table 2-Analysis of multiple regression coefficients between the number of Erythmelus tingitiphagus and meteorological factors temperature $\left({ }^{\circ} \mathrm{C}\right)$ and rainfall (mm), from August 2006 to January 2007 in Itiquira, MT.

\begin{tabular}{cccc}
\hline Coeficientes & Estimativa & $\mathrm{t}$ & $\mathrm{p}$ \\
\hline b0 & $-12,17$ & $-3,19$ & 0,005 \\
b1 & 0,53 & 3,49 & 0,002 \\
b2 & 0,21 & 2,01 & 0,059 \\
b3 & $-0,01$ & $-1,91$ & 0,072 \\
\hline
\end{tabular}

de estratégias de liberação de E. tingitiphagus no campo devem ser incentivados, considerando que esse inimigo natural representa alto potencial de controle de populações de L. heveae em talhões comerciais de seringueira, podendo ser adotado em estratégias de manejo integrado desta importante praga de seringais no Brasil.

\section{CONCLUSÃO}

O pico populacional de Erythmelus tingitiphagus em talhão comercial do clone PB 217 ocorreu no mês de novembro na região, sendo a temperatura e a pluviosidade os principais fatores que afetam a população.

\section{AGRADECIMENTOS}

À empresa “Plantações Edouard Michelin Ltda.", pelo financiamento do projeto, pelo apoio logístico e pela concessão da área experimental; aos seus funcionários Braz da Silva e Silmar Dias Ferreira, pela ajuda na condução dos ensaios; ao Dr. Valmir Antonio Costa (Instituto Biológico/APTA, Campinas, SP), pela identificação do parasitoide; e à tradutora Ariadne Chloe Furnival, pela correção do Abstract.

\section{REFERÊNCIAS}

ALTIERI, M. A. Biodiversity and pest management in Agroecosystems. New York: Haworth Press, 1994. 185p.

ANJOS, N. et al. Árvores e formigas cortadeiras (Hymenoptera: Formicidae) em Viçosa, Minas Gerais. Revista Trópica - Ciências Agrárias e Biológicas, v.2, n.1, p.11-16, 2008.

BELLINI, M. R.; MORAES, G. J.; FERES, R. J. F. Ácaros (Acari) de dois sistemas de cultivo da seringueira no Noroeste do Estado de São Paulo. Neotropical Entomology, v.34, n.3, p.475-484, 2005.

CIVIDANES, F. J.; FONSECA, F. S.; SANTOS, T. M. Distribuição de Leptopharsa heveae em seringal no Estado de São Paulo. Pesquisa Agropecuária Brasileira, v.39, n.10, p.1053-1056, 2004.

COSTA, V. A.; PEREIRA, C. F.; BATISTA FILHO, A. Observações preliminares sobre o parasitismo de ovos de Leptopharsa heveae (Hemiptera: Tingidae) em seringueira em Pindorama, SP. Arquivos do Instituto Biológico, v.70, n.2, p.205-206, 2003. 
COTTA, M. K. et al. Análise econômica do consórcio seringueira-cacau para geração de certificados de emissões reduzidas. Revista Árvore, v.30, n.6, p.969-979, 2006.

FERES, R. J. F. et al. Diversidade de ácaros (Acari, Arachnida) em seringueiras (Hevea brasiliensis Müell. Arg., Euphorbiaceae) na região noroeste do Estado de São Paulo, Brasil.

Revista Brasileira de Zoologia, v.19, n.1, p.137-144, 2002.

FERLA, N. J.; MORAES, G. J. Flutuação populacional e sintomas de dano por ácaros (Acari) em seringueira no estado do Mato Grosso, Brasil. Revista Árvore, v.32, n.2, p.365-376, 2008.

GINGRICH, R. E. Biological control of tephritid fruit flies by inundative releases of natural enemies. In: ALUJA, M.; LIEDO, P. (Ed.). Fruit flies, biology and management. New York: Springer-Verlag, 1993. p.311-318.

GONÇALVES, P. S.; ORTOLANI, A. A.; CARDOSO, M. Melhoramento genético da seringueira: uma revisão. Campinas: Instituto Agronomico de Campinas, 1997. 55p.

(Documentos IAC, 54).

GONÇALVES, P. S. et al. Desempenho de clones de seringueira da série IAC 300 na região do planalto de São Paulo. Pesquisa

Agropecuária Brasileira, v.37, n.2, p.231$138,2002$.

LIVINGSTONE, D.; YACOOB, M. A new subgenus and Epoligosita (Hymenoptera:

Trichogrammatidae) an egg parasite of Tingidae (Heteroptera) from southern India.

Entomophaga, v.28, n.3, p.213-216, 1983.

MAY, A.; GONÇALVES, P. S.; BRIOSCHI, A. P. Consorciação de seringueira com culturas de importância econômica. O Agronômico, v.51, n.1, p.16-23, 1999.

MOREIRA, I. P. S. Biologia da Leptopharsa heveae (Drake \& Poor, 1935) e seus danos nas mudas de Hevea brasiliensis (Müell, 1932). Silvicultura, v.11, n.41, p.47, 1986.
R Development Core Team. R: A language and environment for statistical computing. $R$ Foundation for Statistical Computing, Vienna, Austria. ISBN 3-900051-07-0, URL. Disponível em: <http:// www.R-project.org>. Acesso em: 27 jan. 2013.

RODRIGUES, W. C. Fatores que influenciam no desenvolvimento dos insetos. Info Insetos, v.1, n.4, p.1-4, 2004. Disponível em:

<www.entologistasbrasil.cjb.net $>$ Acesso em: 27 jan. 2013.

RONCHI-TELES, B.; SILVA, N. M. Flutuação populacional de espécies de Anastrepha Schiner (Diptera: Tephritidae) na região de Manaus, AM. Neotropical Entomology, v.34, n.5, p.733$741,2005$.

SCOMPARIN, C. H. J. Estudo dos crisopídeos (Neuroptera, Chrysopidae) em seringueira (Müell. Arg.), aspectos biológicos e potencial no controle biológico de Leptopharsa heveae Drake \& Poor (Hemiptera, Tingidae). 1997. $173 \mathrm{f}$. Dissertação (Mestrado em Agronomia Entomologia Agrícola) - UNESP, Faculdade de Ciências Agrárias e Veterinárias, Jaboticabal, 1997.

SANTOS, R. S. Parasitismo de ovos de Leptopharsa heveae Drake \& Poor, 1935 (Hemiptera: Tingidae) em seringueira (Hevea brasiliensis Müell. Arg.) no estado do Mato Grosso. 2007. 114f. Tese

(Doutorado em Agronomia - Entomologia Agrícola) - UNESP, Faculdade de Ciências Agrárias e Veterinárias, Jaboticabal, 2007.

SANTOS, R. S.; FREITAS, S. Parasitismo de Erythmelus tingitiphagus (Soares)

(Hymenoptera: Mymaridae) em ovos de Leptopharsa heveae Drake \& Poor (Hemiptera: Tingidae), em plantios de seringueira (Hevea brasiliensis Müell. Arg.). Neotropical Entomology, v.37, n.5, p.571-576, 2008a.

SANTOS, R. S.; FREITAS, S. Erythmelus tingitiphagus (Hymenoptera: Mymaridae) um agente promissor no controle biológico do percevejo-de-renda (Hemiptera: Tingidae) da seringueira. Agrotrópica, v.20, n.1, p.25-28, 2008 b.

Revista Árvore, Viçosa-MG, v.37, n.2, p.237-244, 2013 
TANZINI, M. R. Controle do percevejo-de-rendada-seringueira (Leptopharsa heveae) com fungos entomopatogênicos. 2002. 140f. Tese (Doutorado em Entomologia) - Escola Superior de Agricultura "Luiz de Queiroz", Piracicaba, 2002.

TANZINI, M. R.; LARA, F. M. Biologia do percevejo-de-renda-da-seringueira Leptopharsa heveae Drake \& Poor (Heteroptera: Tingidae). Ecossistema, v.23, n.1, p.65-67, 1998.

\section{TROPICOS MISSOURI BOTANICAL GARDEN.}

Disponível em: <http://www.tropicos.org > Acesso em: 27 jan. 2013.

VENDRAMIM, J. D. Praga de viveiros e jardins clonais de seringueira e seu controle. In: MEDRADO, M. J. S. et al. (Ed.). Formação de mudas e plantio de seringueira. Piracicaba: FEALQ, 1992. 158p.
THALER, J. S. Jasmonic acid mediated interactions between plants, herbivores, parasitoids and pathogens: a review of field experiments in tomato. In: AGRAWAL, A. A.; TUZUN, S.; BENT, E. (Ed.). Induced plant defenses against pathogens and herbivores: biochemistry, ecology and agriculture. Saint Paul: American Phytopathological Society Press, 1999. 390p.

WOLDA, H. Seasonality of tropical insects. I. Leafhoppers (Homoptera) in Las Cumbres, Panama. Journal of Animal Ecology, v.49, p.277-290, 1980.

WOLDA, H. Insect seasonality: why? Annual Review of Ecology and Systematics, v.19, p.1-18, 1988. 